\title{
Syndrome of the Trephined: Clinical Spectrum, Risk Factors, and Impact of Cranioplasty on Neurological Recovery in a Prospective Cohort
}

Lukas Sveikata ( $\sim$ lsveikata@mgh.harvard.edu )

Massachusetts General Hospital https://orcid.org/0000-0001-8015-7929

Lana Vasung

Harvard Medical School

Amir El Rahal

Geneva University Hospital

Andrea Bartoli

Geneva University Hospital

Martin Bretzner

Massachusetts General Hospital

Karl Schaller

Geneva University Hospital

Armin Schnider

Geneva University Hospital

Béatrice Leemann

Geneva University Hospital

\section{Research Article}

Keywords: cranioplasty, motor trephine syndrome, postoperative complications, risk factors, sinking skin flap, stroke.

Posted Date: June 4th, 2021

DOl: https://doi.org/10.21203/rs.3.rs-580422/v1

License: (c) (i) This work is licensed under a Creative Commons Attribution 4.0 International License.

Read Full License

Version of Record: A version of this preprint was published at Neurosurgical Review on October 7th, 2021. See the published version at https://doi.org/10.1007/s10143-021-01655-6. 


\section{Abstract}

Background: Syndrome of the Trephined (SoT) is an underrecognized complication after decompressive craniectomy. We aimed to investigate SoT incidence, clinical spectrum, risk factors, and the impact of the cranioplasty on neurological recovery.

Methods: Patients undergoing a large craniectomy $\left(>80 \mathrm{~cm}^{2}\right)$ and cranioplasty were prospectively evaluated using modified Rankin score (mRS) and cognitive (attention, processing speed, executive function, language, visuospatial neglect), motor (Motricity Index, Jamar dynamometer, postural score, gait assessment), and radiological evaluation within four days before and after cranioplasty. The primary outcome was SoT, diagnosed when a neurological improvement was observed after the cranioplasty. The secondary outcome was good outcome (mRS 0-3) four days and 90 days after the cranioplasty. Logistic regression models were used to evaluate the risk factors for SoT and the impact of cranioplasty timing on neurological recovery.

Results: twenty-six patients (65\%) developed SoT and improved after cranioplasty. Brain trauma, hemorrhagic lesions, and shifting of brain structures were associated with SoT. After cranioplasty, a shift towards a good outcome was observed within four days $(p=0.025)$ and persisted at 90 days $(p=0.005)$. Increasing delay to cranioplasty was associated with decreased odds of improvement when adjusting for age and baseline disability (odds ratio $0.96 ; 95 \% \mathrm{Cl}, 0.93-0.99 \mathrm{p}=0.012$ ).

Conclusions: SoT is frequent after craniectomy and interferes with recovery. A high suspicion of SoT should be exercised in patients who fail to progress or have a previous trauma, hemorrhage, or shifting of brain structures. Performing the cranioplasty earlier was associated with improved and quantifiable neurological recovery.

\section{Introduction}

Syndrome of the Trephined (SoT) is an underrecognized complication after decompressive craniectomy (DC) with poorly determined incidence ranging between $1-40 \%$ in the literature.[16, 17, 29, 30, 34, 39] SoT manifests by a delayed sensorimotor or cognitive worsening after craniectomy and is often associated with varying symptomatology, including headache, tinnitus, dizziness, fatigability, pain/discomfort at the site of craniectomy, feeling of apprehension, or depression.[2,15] The wide range of clinical manifestations and absence of well-defined diagnostic criteria makes it a challenging diagnosis in clinical practice. One hallmark feature of SoT is a temporary improvement of symptoms in a supine position, termed orthostatic phenomena, which can help guide the diagnosis, and a definite improvement after the cranioplasty.[5, 22, 35]

Furthermore, SoT is often associated with a sinking skin flap morphology, a radiological[30] and clinical sign [46]. Although this association led to the development of new terminology for the syndrome ('Sinking Skin Flap Syndrome'), numerous findings in the literature indicate the existence of SoT in patients without sinking skin flap morphology.[41] Thus, there is growing evidence that the incidence of SoT might be 
underestimated because of a lack of detailed evaluation of subtle neurological manifestations in the absence of a sinking skin flap. Since DC is increasingly utilized to treat refractory intracranial hypertension for various etiologies, including stroke [28] and traumatic brain injury (TBI)[20], there is an unmet demand for a better understanding of the post-craniectomy related complications.

Although known for almost a century $[15,46]$, the pathophysiology and risk factors predisposing to SoT are largely unknown[2], and as a result, its prediction remains challenging. Furthermore, many patients improve after cranioplasty without previous clinical worsening[19, 37], possibly due to a recovery impediment caused by an insidious and underreported form of SoT. Although emerging evidence suggests that earlier cranioplasty may improve the recovery of neurological function [25], it remains unclear whether the improvement is related to insidious forms of SoT. Diagnostic methods allowing an early diagnosis of SoT and a better risk stratification are needed to inform clinical care management and improve neurological recovery.

To better understand the actual incidence and risk factors of SoT and assess the impact of cranioplasty timing on neurological recovery, we performed a prospective observational study. We aimed to (1) evaluate the neurological function immediately before and after the cranioplasty, (2) compare clinical and radiologic variables between patients with and without SoT, and (3) evaluate the association between the cranioplasty timing and improvement of disability immediately after the procedure.

\section{Materials And Methods}

\section{Design}

From October 2012 to March 2017, we performed a prospective longitudinal cohort study of patients who underwent a large fronto-temporo-parietal DC (axial diameter $>12 \mathrm{~cm}$ ), followed by cranioplasty at Geneva University Hospitals, Geneva, Switzerland. After clinical stabilization, patients transferred to the neurorehabilitation center were consecutively recruited. The participants underwent a comprehensive motor and neurocognitive assessment at admission, within four days before and after cranioplasty, and functional evaluation at 90 days.

The study was approved by the Cantonal Ethics Committee (CCER 11-234) and was performed in accordance with the Declaration of Helsinki. All participants, or their next of kin, when applicable, provided informed consent. The study is in line with STROBE guidelines for observational research.

\section{Participants}

Fifty-one patients referred to the neurorehabilitation center after DC were screened for eligibility. The inclusion criteria were: (1) large DC (axial diameter $>12 \mathrm{~cm}$ ) and (2) age 18 years or more. Exclusion criteria were: (1) refusal to participate $(n=10)$ and (2) immediate severe complication after cranioplasty limiting neurological assessment $(n=1)$. A total of 40 patients were included and completed the 90 days follow-up. 
SoT was defined as neurological deterioration or failure to progress before cranioplasty and a rapid improvement of neurological function within four days after the cranioplasty. Alternative diagnoses were excluded before the cranioplasty, e.g., hydrocephalus, seizure, infection, or new-onset stroke. Based on neurological findings and further clinical workup, two trained neurologists (L.S., B.L.) diagnosed SoT.

\section{Data collection}

Patient demographic, clinical, imaging, and cranioplasty-related complication data were collected. Based on cranioplasty's therapeutic role, we retrospectively stratified groups into 'a priori' and 'a posteriori' SoT. In 'a priori', a more severe form of SoT, patients developed new neurological symptoms or a neurological deterioration before the cranioplasty and improved within four days after the cranioplasty. In 'a posteriori' SoT, patients failed to progress before and improved within four days after the cranioplasty.

\section{Neurological assessment, disability, and imaging biomarkers}

All patients included in the study underwent a comprehensive neurological, including cognitive, assessment at admission, within four days before and four days after the cranioplasty. The neurological assessment included a standard neurological examination and a specific battery of motor tests: Motricity Index[6], grip strength evaluation with Jamar hand dynamometer[4], Postural Assessment Scale for Stroke Patients[3], and qualitative gait independence score ( 0 - bedridden; 1 - walking with substantial assistance from a therapist and $<10 \mathrm{~m}$ distance, 2 - walking with a therapist $>10$-meter distance; 3 independent gait with auxiliary equipment; 4 - independent gait without auxiliary equipment). The cognitive evaluation covered multiple domains, including working memory (digit span forward), executive function and processing speed (Trail Making Test A and B[1], Regard's 5-point non-verbal fluency test[27]), language (institutional 12 object naming test, and Token test[36]), and visuospatial function (Bells cancellation test[13] quantitatively categorized: 0 - no neglect, 1 - mild, 2 - moderate, 3 - severe neglect). The clinical improvement was defined as an improvement of the SoT-related symptoms or improvement in motor/cognitive evaluation performed within four days before and after the cranioplasty. A clinically significant change in neurological function was defined using the reliable change indices $(\mathrm{RCl})$. Using $\mathrm{RCl}$ in psychometric tests allows the determination of whether the change of scores in an individual is significant and is greater than occurring due to random measurement error alone.[10]

The modified Rankin Scale (mRS) was used to measure the degree of disability within four days after the cranioplasty, with mRS 0-3 defining a good outcome. We also recorded the mRS at 90 days to evaluate if the improvement persistent in the long-term period.

All patients underwent a head CT scanner (Siemens Somatom Force or GE Discovery $750 \mathrm{HD}$ ) in a supine position. Imaging biomarkers and standard radiological signs of the SoT were recorded as reported in our previous publication.[41] The following markers indicated a shift of brain structures: the sinking skin flap at the craniectomy site, deviation of the midline structures (figure 1A-D), axial diameter, and slit-like third ventricle and anterior horn of the lateral ventricle. The paradoxical herniation of midline structures was 
marked with positive values, while deviation towards the craniectomy side was marked with negative values.

The craniectomy area was assumed to be an ellipse and calculated using the following equation: $A=(\pi a b) / 4$,

where "a" is the maximal diameter in the axial plane, and "b" is the coronal plane's maximal diameter.

Lastly, we included the radiologic signs of ipsilateral hemorrhagic lesion defined as a composite variable of bleeding in the leptomeningeal compartment either pre- or post-craniectomy procedure: intraparenchymal hemorrhage, subarachnoid hemorrhage, blossoming contusion, or hemorrhagic transformation of ischemic stroke were considered (figure 1B). Subdural and epidural hemorrhages were not included as being outside pial and subarachnoid space they would not affect the perivascular drainage pathways.[42] All measurements were obtained using the Osirix software (Pixmeo Sarl, Geneva, Switzerland).

\section{Statistical analysis}

Continuous variables were compared using Student's t-test or Mann-Whitney $U$ and categorical variables using Fisher's exact or Pearson Chi-square, as appropriate. First, we ran an exploratory univariate analysis to assess baseline imbalances between the SoT vs. non-SoT groups and 'a priori' vs. 'a posteriori' SoT. Exploratory pairwise comparisons were adjusted for false discovery rate (FDR) using the BenjaminiHochberg procedure (FDR <0.1).

Second, to examine the impact of multiple risk factors on SoT, we conducted a forward stepwise logistic regression with three independent variables to avoid overfitting. Variables with a significance of $p<0.1$ in the group comparison analysis were used in univariate analysis; contributing variables with a significance of $p<0.05$ remained in the multivariable model. The explained variance was measured using the Cox \& Snell pseudo- $\mathrm{R}^{2}$ method and the predictive power with the area under the curve (AUC).

Third, the results of individual neurological tests within four days before and four days after the cranioplasty were performed using the t-test and Wilcoxon signed ranks test, as appropriate. On an individual level, a significant improvement on neurological tests was defined using $\mathrm{RCl}$ with $80 \%$ confidence intervals calculated as previously described[38] using the standard deviation of the test and its test-retest reliability index.[36]

Fourth, Cochran's Q test was used for ordinal shift analysis to compare the proportion of good neurological outcomes within four days before and after cranioplasty.

Fifth, the association between disability improvement and delay to cranioplasty was evaluated using a logistic regression model, adjusting for age and baseline disability. $P<0.05$ (2-tailed) was considered 
statistically significant unless stated otherwise. Data analyses were performed using SPSS version 26.0, GraphPad Prism v8.4.0, or R version 3.6.2.

\section{Results}

\section{Cohort characteristics}

Out of 40 patients included in the analysis, $26(65 \%)$ improved motor or cognitive function after the cranioplasty and were diagnosed with SoT. Fourteen patients (35\%) developed clinically appreciable neurological symptoms before the cranioplasty and improved within four days after the cranioplasty ('a priori' SoT). Twelve 12 (30\%) patients presented with failure to progress during the rehabilitation but improved within 4 days after the cranioplasty ('a posteriori' SoT). The mean delay to cranioplasty was $112.8 \pm 35.4$ days. Table 1 presents the univariate comparison of clinical and imaging characteristics between SoT and non-SoT groups. There was a strong association between SoT and ipsilateral hemorrhagic lesions $(p=0.004)$ and shifting of brain structure $(p<0.001)$. There was also a weak association between SoT and TBI and an inverse association with ischemic stroke, although it did not maintain statistical significance after the FDR correction. Two patients, both in the SoT group, required a placement of ventriculoperitoneal drain for post-traumatic hydrocephalus prior to cranioplasty. The material used for cranioplasty did not differ between the groups and was autologous bone in 33 (82.5\%), polyetheretherketone implant in $6(15 \%)$, and titanium plating in $1(2.5 \%)$.

\section{Risk factors}

The unadjusted logistic regression model identified that TBI, ipsilateral hemorrhagic lesions, and shifting of brain structures were significantly associated with SoT (Table 2). In the multivariable regression model adjusted for age, ipsilateral hemorrhagic lesions, and shifting of brain structures remained strong independent predictors of SoT, while TBI maintained a weak association with SoT. The model explained a significant proportion of the SoT occurrence variability (Cox \& Snell $\left.R^{2}=0.45\right)$ and had a strong predictive power (AUC $=0.93$ ).

\section{Key features of SoT}

When comparing the two modes of SoT presentation, the 'a posteriori' SoT individuals were younger (38.8 \pm 10.2 vs. $52.5 \pm 11.8$ years, $p=0.004$ ) but did not differ in other clinical and demographic characteristics. The 'a posteriori' SoT presented with less prominent radiological features. Sinking skin flap ( $50 \%$ vs. $93 \%$, $p=0.026)$ and paradoxical herniation $(17 \%$ vs. $64 \%, p=0.021)$ were less common in the 'a posteriori' SoT group but did not differ in other radiological features.

\section{Outcomes after the cranioplasty}

Neurological improvement 
All 26 SoT patients presented a rapid motor, cognitive improvement, or improvement in SoT symptoms (orthostatic phenomena, headache, vertigo, etc.) within four days after the cranioplasty (Table 3).

The baseline performance in motor and cognitive tests four days before the cranioplasty did not differ significantly between the SoT and non-SoT groups. However, there was significant improvement in motor and cognitive performance in the SoT group within 4 days after the cranioplasty (Table 4). SoT patients improved significantly in Motricity Index, postural balance (PASS), gait, executive function (TMT A test and Regard's 5-point verbal fluency test), and spatial neglect performance. Among 'a priori' SoT patients, the cognitive and motor recovery was complete in 6/14 (43\%) and partial in 8/14 (57\%).

\section{Change in disability}

The neurological improvement resulted in an improved disability (mRS) within four days after the cranioplasty in 7/26 (27\%) SoT patients. Cochran's Q test indicated a significant ordinal shift towards a good outcome after the cranioplasty ( $58 \%$ vs. $45 \%, p=0.025$; figure $2 \mathrm{~A}$ ).

In the SoT group, there was a significant shift towards a good outcome within four days after the cranioplasty $(p=0.025)$ that was maintained at 90 days $(p=0.005$, figure $2 B)$.

In the 'a priori' SoT group, a similar shift towards good outcome $(p=0.025)$ and improved mRS $(p=$ 0.006) was observed. In the 'a posteriori' SoT group, the neurological improvement did not change the mean mRS within four days after the cranioplasty. Nevertheless, there was a trend towards a good outcome $(p=0.157)$ and a significant mean mRS improvement at 90 days $(p=0.007)$ that was not observed in the non-SoT group.

\section{Delay to Cranioplasty and Neurological Recovery}

We observed a significant delay between the resolution of brain swelling at the craniectomy site (54.3 \pm 40.4 days) and the delay to cranioplasty (112.8 \pm 35.4 ; figure 3$)$. The delay from edema disappearance to cranioplasty was longer than one month in $30(75 \%)$ and longer than two months in 18 (45\%) study participants.

Increasing delay to cranioplasty was independently associated with less neurological improvement. The odds for improvement decreased by $4 \%$ for every additional day to cranioplasty after adjusting for age and baseline disability ( $\mathrm{OR}=0.96,95 \% \mathrm{Cl} 0.93-0.99 ; \mathrm{p}=0.026$; figure 4). An additional delay of 30 days decreased the odds to improve by $29 \%$. No improvement was observed when the delay to cranioplasty was 135 days or longer.

\section{Discussion}

The main finding of this study is the high incidence of SoT in patients with large craniectomies, suggesting that the condition is underreported. $[16,17,30,34,39]$ Contrary to previous studies we have identified that $65 \%$ patients suffered from SoT. A detailed prospective neurologic and radiologic 
assessment within four days before and after cranioplasty, allowed to identify the spectrum of SoT condition. We then identified three independent risk factors contributing to SoT that may help stratify SoT risk in future studies. Third, we identified and quantified the impact of cranioplasty timing on neurological recovery. Our findings suggest an association between earlier cranioplasty and improved neurological recovery.

\section{Detailed neurological assessment is instrumental in detecting SoT}

A detailed neurological and cognitive assessment within four days before and after the cranioplasty allowed to detect the milder 'a posteriori' SoT cases. A posteriori SoT manifest by slow rehabilitation progress and presented an objective neurological improvement within four days after the cranioplasty. The improved diagnostic sensitivity explains a significantly higher incidence of SoT in our cohort. Our study adds to the growing body of literature demonstrating that a significant proportion of patients improve after cranioplasty even without evident neurological worsening beforehand.[19] Our findings showing an improvement of neurological function in the 'a posteriori' SoT group corroborate the observations that a proportion of patients improve after cranioplasty even without an apparent worsening beforehand. Thus, our findings suggest that 'a priori' and 'a posteriori' SoT constitute a spectrum of clinical severity of SoT. Consequently, a significant proportion of patients after craniectomy is susceptible to develop SoT, and the lack of rehabilitation progress in craniectomized patients should alert clinicians to consider tailoring the cranioplasty timing to an individual patient.

The SoT diagnosis remains challenging due to the absence of robust diagnostic criteria. In the current study, the radiological assessment revealed that $81 \%$ of the SoT group presented at least one sign of shifting brain structures, e.g. sinking skin flap, paradoxical midline shift, compressed lateral or $3^{\text {rd }}$ ventricle, but diagnostic yield individually remained low. SoT manifested without the classical radiological sign of sinking skin flap in $50 \%$ and without paradoxical herniation in more than $80 \%$ of the 'a posteriori' SoT patients, corroborating our previous study.[41] As a result, the absence of a sinking skin flap or paradoxical herniation does not exclude SoT, and careful clinical evaluation should guide the diagnosis. Conversely, radiologic signs of the brain structure shifting should warrant a careful repeated neurological evaluation and to consider an expedited cranioplasty.

\section{Brain injury, hemorrhage, and shifting of the brain are risk factors for SoT}

Our results suggest that hemorrhagic lesions in the leptomeningeal compartment and shifting of brain structures are strongly associated with the development of SoT. Although TBI did not maintain a strong association in multivariable analysis, the association between TBI and SoT could be clinically significant, warranting confirmation in larger cohorts.

There was no significant confounding between the shift of brain structures and TBI or the hemorrhagic lesions and TBI. Consequently, we suggest a cumulative effect of these three risk factors on the development of SoT. Stiver et al. showed that brain contusions, together with abnormal cerebrospinal fluid (CSF) circulation, represent a risk factor for developing SoT.[34] Similarly, in a study evaluating a 
large DC registry including 43 SoT cases Di Rienzo et al. found an association between TBI and SoT.[29] On the other hand, although our results suggest that ischemic stroke presents a lower risk for SoT, stroke etiology should not exclude SoT, as it has been previously shown to occur in stroke cohorts.[30]

\section{The three-hit hypothesis for developing SoT}

Based on the study results, we suggest a three-hit hypothesis for the pathophysiology of SoT:

- Initial brain injury by TBI causes a wide range of functional short- and long-term neurological deficits associated with contusions[33] and diffuse axonal injury[32] with underlying healthy brain tissue that has the potential to recover.

- Cranial window results in brain structure shift and disturbs physiologic intracranial fluid dynamics. The physical shift of brain structures and compression by a sinking skin flap causes blood flow disturbances,[43] cerebral metabolism impairment[43, 47], and changes in the CSF flow[11, 41]. The loss of the brain's rigid enclosing causes reduced pulse wave amplitude[24], which impairs intracranial fluid movement, including capillary blood flow, CSF circulation, and perivascular drainage[26].

- Hemorrhagic lesions further impair CSF production and clearance. Increased atmospheric pressure and blood degradation products in the brain parenchyma[12] and subarachnoid space[14] impair CSF formation and clearance through blockade of arachnoid granulations by blood clots. Additionally, emerging evidence on the role of perivascular drainage of brain solutes and its impairment may additionally contribute to impaired brain fluids dynamics. [12, 23]

Interestingly, we did not find association between craniectomy size and SoT. Recent studies have shown an association between craniectomy size and SoT.[29, 39] In a study by Tarr et al. SoT incidence increased sharply when craniectomy area reached $50 \mathrm{~cm}^{2}$ and beyond[39]. Although we did not find a difference in mean craniectomy area between SoT and non-SoT group, the inclusion of only large craniectomies in our study (mean craniectomy area $112.8 \pm 35.4 \mathrm{~cm}^{2}$ ) limited the power to detect this association. Nevertheless, large craniectomies may have contributed to the higher incidence of SoT in our cohort.

\section{Effect of cranioplasty on neurological symptoms and disability}

All SoT patients improved in motor and cognitive function within four days after the cranioplasty confirming the SoT diagnosis.[2] In contrast, Honeybul et al. reported a 16\% improvement rate. However, their cohort did not include patients with worsening neurological status before the cranioplasty (i.e., 'a priori' SoT), arguably representing less severe SoT cases. According to the literature, the delay to improvement observed after cranioplasty varies from 1-4 days. $[2,17,39]$ There is also evidence suggesting that cerebral perfusion abnormalities improve in a similar timeframe.[35] As a result, evaluating neurological symptoms within four days in this study was considered optimal for increasing the SoT detection sensitivity. 
Our results revealed that neurological improvement after cranioplasty in SoT patients led to a significant improvement in the quality of life (i.e., decreased disability) and a shift towards good neurological outcome within four days after the cranioplasty. This study reports a clear and measurable improvement in an individual's quality of life after the cranioplasty and adds to the growing body of literature on the impact of cranioplasty on neurological recovery.

\section{Does timing of the cranioplasty improve neurological recovery?}

We found an association between improved neurological recovery and shorter delays to cranioplasty. Although our results are in line with the emerging evidence that cranioplasty may improve neurological function, and earlier cranioplasty may enhance this effect [18, 25], the question of optimal timing for cranioplasty procedure remains a complex issue. Multiple factors are at play when determining the optimal timing. One of them, addressed in our study, is the resolution of brain swelling giving place to restore the skull integrity successfully.

Our study results suggest a potential window to perform cranioplasty as early as two weeks after the craniectomy as soon as the brain swelling resides. Potential benefits from cranioplasty, such as improved postural blood flow[43], cerebrovascular reserve capacity, cerebral metabolism[43, 47], and improvement in CSF flow[9], offer compelling arguments in favor of an earlier cranioplasty. In line with these previous studies, intracranial ICP measurements showed that physiological ICP dynamics during changes from supine to vertical position were restored after the cranioplasty.[24] Thus, earlier cranioplasty could improve the aptitude to perform rehabilitation activities in a seated or standing position. However, to fully understand the equation for optimal cranioplasty timing, multiple variables must be considered, such as the cranioplasty-related complications[31], risk of infection[40], role of post-traumatic hydrocephalus[21], over-drainage related to ventriculoperitoneal shunting [21], pre-cranioplasty morbidity[7], bleeding diathesis, and conditions related to the initial etiology for a craniectomy.

The evidence for complication risk regarding the timing of cranioplasty is conflicting. In our clinical practice, cranioplasty is often performed three or more months after DC.[44, 45] Some authors have suggested that delaying the cranioplasty beyond two or even six months after DC might reduce the risk of complications and surgical site infections.[31, 40] Contrary to these findings, a meta-analysis of 18 studies and 2254 patients did not show any difference in infection rates when cranioplasty was performed earlier than three months after DC.[45] Another meta-analysis of 1209 patients confirmed similar infectious and hemorrhagic complication rates regardless of timing.[44] However, they also found an increased risk of hydrocephalus in the early cranioplasty group (relative risk $2.67,95 \% \mathrm{Cl} 1.24-5.73$ ), highlighting the complex relation of CSF circulation and the timing of cranioplasty.[44]

In addition, the time of onset of 'a posteriori' SoT cannot be fully appreciated as this insidious form of SoT rarely presents the 'red flags', such as sinking skin flap, paradoxical herniation, orthostatic phenomena, headaches, vertigo, etc. Our findings suggest that some of the SoT symptoms might not be fully reversible, further adding to the argument in favor of an earlier cranioplasty. In line, with our findings in previous studies the proportion of complete recovery ranged from $34.6 \%$ to $78 \%[2,29,39]$ and the 
improvement continued up to 7 weeks [39]. It is possible that earlier cranioplasty could prevent SoT but it remains unclear if earlier cranial may be beneficial in all craniectomized patients or a subgroup with higher SoT risk. Performing cranioplasty as soon as brain tissue edema resolves may be preferable as it could improve participation in rehabilitation[8] and neurological outcome.[18, 25] Because of the varying delay to brain swelling resolution and growing observational evidence of neurological improvement after cranioplasty[25], future studies should seek to risk stratify patients and tailor the timing of the cranioplasty to an individual patient rather than perform it at a fixed delay.

The reasons for the increased delay between brain edema resolution and cranioplasty in our study are not clear. However, some of the contributing factors might be administrative and logistical considerations. It is crucial to foresee a timely transfer to a neurosurgical center when one is not available in the rehabilitation center's vicinity. Secondly, some delays may occur due to the logistical delays required to produce and deliver a personalized cranial flap, such as using the custom-made polyetheretherketone flaps. Thus, efforts should be made to establish an early collaboration between the rehabilitation and neurosurgical teams to streamline the logistical aspects of the cranioplasty procedure.

\section{Limitations}

The relatively small sample size resulted in wide confidence intervals, signifying a low level of precision, and should be interpreted with caution. Due to the increased number of univariate analyses, we ran into the risk of type I error due to multiple comparisons that we adjusted for using FDR corrections. Hence, our results should be regarded as hypothesis-generating, highlighting potential mechanisms and associations to be confirmed in future studies on SoT. Nevertheless, it is reasonable to provide an informed discussion based on these pathophysiological hypotheses and assumptions in an effort to build a model or apply advanced neuroimaging techniques such as perfusion-weighted imaging or glymphatic MRI, which could further explain clinical and research findings and help develop hypothesisdriven studies in the field. More imaging and histopathological studies are needed to unravel the mechanisms of SoT that could lead to improved neurological recovery in this fragile patient population.

Due to the relatively small sample size and observational design, we could not control for multiple variables influencing the delay to cranioplasty. Our findings, although preliminary, suggest an association between earlier cranioplasty and improved neurological recovery. Randomized trials with larger sample sizes are warranted[21] to explore this association, further controlling for multiple confounding factors and effect modifiers. Furthermore, cranioplasty is known to carry a high risk of postoperative complications, and therefore, post-operative hemorrhagic complications might mask the effect of cranioplasty on neurological improvement. Lastly, ten patients failed to consent, potentially contributing to consent bias, which is relatively low due to the random distribution of non-consenting patients.

\section{Conclusion}

This prospective longitudinal study demonstrates that SoT manifests with a spectrum of cognitive and motor symptoms in more than half of the patients undergoing craniectomy, causing significant concern 
for neurorehabilitation. Neurological deterioration or a lack of rehabilitation progress in craniectomized patients should alert clinicians to consider SoT. Our findings suggest that ipsilateral hemorrhagic lesions, shifting of brain structures, and brain lesions related to TBI, are independently associated with the pathophysiology of SoT. A cranioplasty performed as soon as brain edema resolves could help prevent the development of SoT and improve neurological recovery, but further studies are needed to determine the optimal timing for cranial repair.

\section{Abbreviations}

SoT $=$ syndrome of the trephined

$\mathrm{DC}=$ decompressive craniectomy

$\mathrm{TBI}=$ traumatic brain injury

CSF $=$ cerebrospinal fluid

$\mathrm{mRS}=$ modified Rankin scale

\section{Declarations}

Funding: This study was in part supported by a scholarship from the Swiss National Science Foundation (P2GEP3_191584 (L.S.)), statistics consultations from Harvard Catalyst I The Harvard Clinical and Translational Science Center (National Center for Advancing Translational Sciences, National Institutes of Health Award UL 1TR002541) and financial contributions from Harvard University and its affiliated academic healthcare centers. The content is solely the responsibility of the authors and does not necessarily represent the official views of Harvard Catalyst, Harvard University and its affiliated academic healthcare centers, or the National Institutes of Health.

Conflicts of interest: The authors declare that they have no conflict of interest.

Availability of data and material: The data that support the findings of this study are available from the corresponding author, upon reasonable request.

Code availability: Not applicable.

Ethical approval: The study was approved by the Cantonal Ethics Committee (CCER 11-234). The study procedures were performed in accordance with the Declaration of Helsinki and its later amendments or comparable ethical standards.

Consent to participate: All participants, or their next of kin, when applicable, provided informed consent.

Consent for publication: Not applicable. 
Authors' Contributions: Conceptualization: Béatrice Leemann, Armin Schnider, Lukas Sveikata; Methodology: Lukas Sveikata, Beatrice Leemann; Formal analysis and investigation: Lukas Sveikata; Writing - original draft preparation: Lukas Sveikata; Writing - review and editing: Lana Vasung, Amir El Rahal, Andrea Bartoli, Martin Bretzner, Karl Schaller, Armin Schnider, Béatrice Leemann, Resources: Armin Schnider, Beatrice Leemann; Supervision: Armin Schnider, Beatrice Leemann.

\section{Acknowledgments}

We thank the participants and their families for taking part in the study. We sincerely acknowledge the neurorehabilitation center staff for helping perform the study and for the care of the study participants.

\section{References}

1. Arbuthnott K, Frank J (2000) Trail Making Test, Part B as a Measure of Executive Control: Validation Using a Set-Switching Paradigm. J Clin Exp Neuropsychology Neuropsychology Dev Cognition Sect 22(4):518-528

2. Ashayeri K, Jackson EM, Huang J, Brem H, Gordon CR (2016) Syndrome of the Trephined: A Systematic Review. Neurosurgery 79(4):525-534

3. Benaim C, Pérennou DA, Villy J, Rousseaux M, Pelissier JY (1999) Validation of a Standardized Assessment of Postural Control in Stroke Patients. Stroke 30(9):1862-1868

4. Bertrand AM, Fournier K, Brasey M-GW, Kaiser M-L, Frischknecht R, Diserens K (2015) Reliability of maximal grip strength measurements and grip strength recovery following a stroke. J Hand Ther 28(4):356-363

5. Bijlenga P, Zumofen D, Yilmaz H, Creisson E, Tribolet N de (2007) Orthostatic mesodiencephalic dysfunction after decompressive craniectomy. J Neurology Neurosurg Psychiatry 78(4):430

6. Collin C, Wade D (1990) Assessing motor impairment after stroke: a pilot reliability study. J Neurology Neurosurg Psychiatry 53(7):576

7. Coulter IC, Pesic-Smith JD, Cato-Addison WB, Khan SA, Thompson D, Jenkins AJ, Strachan RD, Mukerji N (2014) Routine but risky: A multi-centre analysis of the outcomes of cranioplasty in the Northeast of England. Acta Neurochir 156(7):1361-1368

8. Dujovny M, Aviles A, Agner C, Fernandez P, Charbel FT (1997) Cranioplasty: Cosmetic or therapeutic? Surg Neurol 47(3):238-241

9. Dujovny M, Fernandez P, Alperin N, Betz W, Misra M, Mafee M (1997) Post-cranioplasty cerebrospinal fluid hydrodynamic changes: Magnetic resonance imaging quantitative analysis. Neurol Res 19(3):311-316

10. Evans C, Margison F, Barkham M (1998) The contribution of reliable and clinically significant change methods to evidence-based mental health. Évid Based Ment Heal 1(3):70 72

11. Fodstad H, Love JA, Ekstedt J, Frid $>n$ H, Liliequist B (1984) Effect of cranioplasty on cerebrospinal fluid hydrodynamics in patients with the syndrome of the trephined. Acta Neurochir 70(1-2):21-30 
12. Gaberel T, Gakuba C, Goulay R, Lizarrondo SMD, Hanouz J-L, Emery E, Touze E, Vivien D, Gauberti M (2014) Impaired Glymphatic Perfusion After Strokes Revealed by Contrast-Enhanced MRI: A New Target for Fibrinolysis? Stroke 45(10):3092-3096

13. Gauthier L, Dehaut F, Joanette Y (1989) The Bells Test: A quantitative and qualitative test for visual neglect. International Journal of Clinical Neuropsychology 2(11):49-54

14. Goulay R, Flament J, Gauberti M, et al (2017) Subarachnoid Hemorrhage Severely Impairs Brain Parenchymal Cerebrospinal Fluid Circulation in Nonhuman Primate. Stroke 48(8):2301-2305

15. Grant FC, Norcross NC (1939) Repair of cranial defects by cranioplasty. Ann Surg 110(4):488-512

16. Honeybul S (2010) Complications of decompressive craniectomy for head injury. J Clin Neurosci $17(4): 430-435$

17. Honeybul S, Ho KM (2011) Long-Term Complications of Decompressive Craniectomy for Head Injury. J Neurotraum 28(6):929-935

18. Honeybul S, Janzen C, Kruger K, Ho KM (2013) The impact of cranioplasty on neurological function. Brit J Neurosurg 27(5):636-641

19. Honeybul S, Janzen C, Kruger K, Ho KM (2016) The Incidence of Neurologic Susceptibility to a Skull Defect. World Neurosurg 86:147-152

20. Hutchinson PJ, Kolias AG, Timofeev IS, et al (2016) Trial of Decompressive Craniectomy for Traumatic Intracranial Hypertension. New Engl J Medicine 375(12):1119-1130

21. Iaccarino C, Kolias A, Adelson PD, et al (2020) Consensus statement from the international consensus meeting on post-traumatic cranioplasty. Acta Neurochir 1-18

22. Joseph V, Reilly P (2009) Syndrome of the trephined. J Neurosurg 111(4):650-652

23. Klarica M, Radoš M, Orešković D (2019) The Movement of Cerebrospinal Fluid and Its Relationship with Substances Behavior in Cerebrospinal and Interstitial Fluid. Neuroscience 414:28-48

24. Lilja-Cyron A, Andresen M, Kelsen J, Andreasen TH, Petersen LG, Fugleholm K, Juhler M (2019) Intracranial pressure before and after cranioplasty: insights into intracranial physiology. J Neurosurg $1-11$

25. Malcolm JG, Rindler RS, Chu JK, Chokshi F, Grossberg JA, Pradilla G, Ahmad FU (2017) Early Cranioplasty is Associated with Greater Neurological Improvement: A Systematic Review and MetaAnalysis. Neurosurgery 82(3):278-288

26. Mestre H, Mori Y, Nedergaard M (2020) The Brain's Glymphatic System: Current Controversies. Trends Neurosci 43(7):458-466

27. Regard M, Strauss E, Knapp P (1982) Children's Production on Verbal and Non-Verbal Fluency Tasks. Percept Motor Skill 55(3):839-844

28. Reinink H, Jüttler E, Hacke W, et al (2021) Surgical Decompression for Space-Occupying Hemispheric Infarction. Jama Neurol. doi: 10.1001/jamaneurol.2020.3745

29. Rienzo AD, Colasanti R, Gladi M, Pompucci A, Costanza MD, Paracino R, Esposito D, lacoangeli M (2020) Sinking flap syndrome revisited: the who, when and why. Neurosurg Rev 43(1):323-335 
30. Sarov M, Guichard J-P, Chibarro S, Guettard E, Godin O, Yelnik A, George B, Bousser M-G, Vahedi K, investigators the D (2010) Sinking Skin Flap Syndrome and Paradoxical Herniation After Hemicraniectomy for Malignant Hemispheric Infarction. Stroke 41(3):560-562

31. Schuss P, Vatter H, Marquardt G, Imöhl L, Ulrich CT, Seifert V, Güresir E (2012) Cranioplasty after Decompressive Craniectomy: The Effect of Timing on Postoperative Complications. J Neurotraum 29(6):1090-1095

32. Sidaros A, Engberg AW, Sidaros K, Liptrot MG, Herning M, Petersen P, Paulson OB, Jernigan TL, Rostrup E (2008) Diffusion tensor imaging during recovery from severe traumatic brain injury and relation to clinical outcome: a longitudinal study. Brain 131(2):559-572

33. Soustiel JF, Mahamid E, Goldsher D, Zaaroor M (2007) Perfusion-CT for early assessment of traumatic cerebral contusions. Neuroradiology 50(2):189-196

34. Stiver SI, Wintermark M, Manley GT (2008) Reversible monoparesis following decompressive hemicraniectomy for traumatic brain injury. J Neurosurg 109(2):245-54

35. Stiver SI, Wintermark M, Manley GT (2008) Motor trephine syndrome: A mechanistic hypothesis. 273-277

36. Strauss E, Sherman EMS, Spreen O (2006) A compendium of neuropsychological tests: Administration, norms, and commentary, 3rd ed. Oxford University Press, New York, NY, US

37. Suzuki N, Suzuki S, Iwabuchi T (1993) Neurological improvement after cranioplasty. Acta Neurochir $122(1-2): 49-53$

38. Sveikata L, Kavan N, Pegna AJ, Seeck M, Assal F, Momjian S, Schaller K, Vulliemoz S (2019) Postoperative memory prognosis in temporal lobe epilepsy surgery: The contribution of postictal memory. Epilepsia. doi: 10.1111/epi.16281

39. Tarr JT, Hagan M, Zhang B, Tanna N, Andrews BT, Lee JC, Bradley JP (2020) Syndrome of the Trephined: Quantitative Functional Improvement after Large Cranial Vault Reconstruction. Plast Reconstr Surg 1

40. Thavarajah D, Lacy PD, Hussien A, Sugar A (2011) The minimum time for cranioplasty insertion from craniectomy is six months to reduce risk of infection- a case series of 82 patients. Brit J Neurosurg 26(1):78-80

41. Vasung L, Hamard M, Soto MCA, Sommaruga S, Sveikata L, Leemann B, Vargas MI (2016) Radiological signs of the syndrome of the trephined. Neuroradiology 58(6):557 568

42. Wardlaw JM, Benveniste H, Nedergaard M, et al (2020) Perivascular spaces in the brain: anatomy, physiology and pathology. Nat Rev Neurology 16(3):137-153

43. Winkler PA, Stummer W, Linke R, Krishnan KG, Tatsch K (2000) Influence of cranioplasty on postural blood flow regulation, cerebrovascular reserve capacity, and cerebral glucose metabolism. J Neurosurg 93(1):53-61

44. Xu H, Niu C, Fu X, Ding W, Ling S, Jiang X, Ji Y (2015) Early cranioplasty vs. late cranioplasty for the treatment of cranial defect: A systematic review. Clin Neurol Neurosur 136:33-40 
45. Yadla S, Campbell PG, Chitale R, Maltenfort MG, Jabbour P, Sharan AD (2011) Effect of Early Surgery, Material, and Method of Flap Preservation on Cranioplasty InfectionsA Systematic Review. Neurosurgery 68(4):1124-1130

46. Yamaura A, Makino H (1977) Neurological Deficits in the Presence of the Sinking Skin Flap following Decompressive Craniectomy. Neurol Med-chir 17pt1(1):43-53

47. Yoshida K, Furuse M, Izawa A, lizima N, Kuchiwaki H, Inao S (1996) Dynamics of cerebral blood flow and metabolism in patients with cranioplasty as evaluated by $133 \mathrm{Xe} \mathrm{CT}$ and $31 \mathrm{P}$ magnetic resonance spectroscopy. J Neurology Neurosurg Psychiatry 61(2):166-171

\section{Tables}

Table 1. Clinical and imaging characteristics 


\begin{tabular}{|c|c|c|c|c|}
\hline Characteristics & $\begin{array}{l}\text { All cohort } \\
(n=40)\end{array}$ & $\begin{array}{l}\text { SoT } \\
(n=26)\end{array}$ & $\begin{array}{l}\text { Non-SoT } \\
(n=14)\end{array}$ & Palue* \\
\hline \multicolumn{5}{|l|}{ Demographics and clinical } \\
\hline Male, $\mathrm{n}(\%)$ & $26(65)$ & $17(65)$ & $9(64)$ & 1.000 \\
\hline Age, y $($ mean $\pm S D)$ & $46.2 \pm 12.6$ & $46.2 \pm 12.9$ & $46.3 \pm 12.5$ & 0.983 \\
\hline Craniectomy area, $\mathrm{cm} 2($ mean $\pm \mathrm{SD})$ & $\begin{array}{l}115.7 \pm \\
18.5\end{array}$ & $\begin{array}{l}114.0 \pm \\
17.4\end{array}$ & $\begin{array}{l}118.9 \pm \\
20.6\end{array}$ & 0.428 \\
\hline Time to cranioplasty, days & $\begin{array}{l}112.8 \pm \\
35.4\end{array}$ & $\begin{array}{l}107.8 \pm \\
41.0\end{array}$ & $\begin{array}{l}122.1 \pm \\
19.9\end{array}$ & 0.146 \\
\hline Left craniectomy, n (\%) & $19(48)$ & $15(58)$ & $4(29)$ & 0.105 \\
\hline Post-traumatic hydrocephalus, n (\%) & $2(5)$ & $2(8)$ & 0 & 0.533 \\
\hline \multicolumn{5}{|l|}{ Etiology for craniectomy, n (\%) } \\
\hline TBI & $15(38)$ & $13(50)$ & $2(14)$ & 0.040 \\
\hline Ischemic stroke & $13(33)$ & $5(19)$ & $8(57)$ & 0.031 \\
\hline Hemorrhagic stroke & $8(20)$ & $6(23)$ & $2(14)$ & 0.689 \\
\hline SAH & $1(3)$ & 0 & $1(7)$ & 0.350 \\
\hline Other & $3(8)$ & $2(8)$ & $1(7)$ & 1.000 \\
\hline \multicolumn{5}{|l|}{ Imaging } \\
\hline Ipsilateral hemorrhagic lesions, n (\%) & $31(88)$ & $24(92)$ & $7(50)$ & $0.004^{*}$ \\
\hline Shifting of brain structures, $\mathrm{n}(\%)$ & $24(60)$ & 210 & $3(21)$ & $<0.001 *$ \\
\hline Sinking skin flap, n (\%) & $22(55)$ & $19(73)$ & $3(21)$ & $0.003^{*}$ \\
\hline Midline shift, mm (mean \pm SD) & $0.1 \pm 3.8$ & $1.2 \pm 3.9$ & $-1.8 \pm 2.8$ & $0.017 *$ \\
\hline Paradoxical herniation, n (\%) & $13(33)$ & $11(42)$ & $2(14)$ & 0.090 \\
\hline $\begin{array}{l}3 \mathrm{rd} \text { ventricle axial diameter, } \mathrm{mm} \text { (mean } \pm \\
\mathrm{SD} \text { ) }\end{array}$ & $5.8 \pm 3.4$ & $5.3 \pm 3.7$ & $6.8 \pm 2.5$ & 0.073 \\
\hline Slit 3rd ventricle, n (\%) & $16(40)$ & $14(54)$ & $2(14)$ & $0.020^{*}$ \\
\hline AHLV diameter, mm (mean $\pm \mathrm{SD})$ & $12.1 \pm 6.8$ & $10.9 \pm 7.5$ & $14.2 \pm 4.6$ & 0.103 \\
\hline Slit AHLV, n (\%) & $15(38)$ & $14(54)$ & $1(7)$ & $0.005^{*}$ \\
\hline \multicolumn{5}{|l|}{ Cranioplasty complications, $\mathrm{n}(\%)$} \\
\hline Hemorrhagic complication & $18(45)$ & $12(46)$ & $6(43)$ & 0.842 \\
\hline Requiring surgery & $11(28)$ & $7(27)$ & $4(29)$ & 1.000 \\
\hline
\end{tabular}


* Significant values after FDR correction are highlighted. Abbreviations: SoT, syndrome of the trephined; $\mathrm{OR}$, odds ratio; $\mathrm{Cl}$, confidence interval; $\mathrm{SD}$, standard deviation; $\mathrm{TBI}$, traumatic brain injury; SAH, subarachnoid hemorrhage; mRS, modified Rankin scale; ICU, intensive care unit; AHLV, anterior horn of lateral ventricle; FDR, false discovery rate.

Table 2. Logistic regression for SoT prediction

\begin{tabular}{|c|c|c|c|c|c|c|}
\hline \multirow[b]{2}{*}{ Variable } & \multicolumn{3}{|c|}{ Unadjusted } & \multicolumn{3}{|c|}{ Adjusted $^{b}$} \\
\hline & OR & $95 \% \mathrm{Cl}$ & p Value & OR & $95 \% \mathrm{Cl}$ & p Value \\
\hline Traumatic brain injury & 6.0 & $3.1-76.8$ & 0.03 & 9.9 & $0.9-112.2$ & 0.065 \\
\hline Ipsilateral hemorrhagic lesion & 12.0 & $2.0-71.4$ & 0.006 & 22.1 & $1.4-354.0$ & 0.029 \\
\hline Shifting of brain structures ${ }^{b}$ & 15.4 & $3.1-76.8$ & $<0.001$ & 13.9 & $2.0-97.6$ & 0.008 \\
\hline \multicolumn{7}{|c|}{$\begin{array}{l}\text { Multivariable regression performed by stepwise method. Variance explained by } R^{2}=0.45 \text { (Cox \& } \\
\text { Snell), AUC }=0.93 . \text { Model } \chi^{2}(1)=24.14 \text {. Abbreviations: SoT, Syndrome of the Trephined, OR, odds } \\
\text { ratio, Cl, confidence interval, AUC, are under the curve. }\end{array}$} \\
\hline
\end{tabular}

Table 3. Key clinical features of SoT 


\section{Characteristic}

SoT cases

$(n=26)$

Delay to key events, days (mean \pm SD)

DC to brain swelling resolution

$45.9 \pm 35.4$

$D C$ to SoT ${ }^{a}$

$64.8 \pm 24.8$

DC to cranioplasty

$107.8 \pm 41.0$

Motor/sensitive, n (\%)

$21(81)$

Motor impairment

$21(81)$

Gait disturbance

$6(23)$

Pyramidal signs

$3(12)$

Sensitive deficit

$1(4)$

Cortical functions, n (\%)

$23(88)$

Executive function and attention

$14(54)$

Hemineglect

10 (39)

Language deficit

10 (39)

Altered mental state

4 (15)

Visual disturbance

3 (12)

Other symptoms, n (\%)

Orthostatic phenomena

$12(46)$

Headache

5 (19)

Nausea and vomiting

$3(12)$

Seizures

3 (12)

Fatigue

2 (8)

Vertigo

1 (4)

a - only cases where objective neurological deterioration was identified before the cranioplasty $(n=14)$. Values are mean $\pm S D, n(\%)$ where appropriate. Abbreviations: $D C$, decompressive craniectomy, SoT, syndrome of the trephined. 
Table 4. Comparison of motor and cognitive performance in SoT patients 4 days before and after the cranioplasty

\begin{tabular}{|c|c|c|c|c|}
\hline Evaluation in SoT group & $\mathbf{n}$ & $\begin{array}{l}\text { Before } \\
\text { cranioplasty }\end{array}$ & $\begin{array}{l}\text { After } \\
\text { cranioplasty }\end{array}$ & $\begin{array}{l}\text { p } \\
\text { Value }^{a}\end{array}$ \\
\hline \multicolumn{5}{|l|}{ Motor function } \\
\hline Motricity Index, mean \pm SD & 20 & $37.9 \pm 30.0$ & $51.1 \pm 35.5$ & 0.024 \\
\hline Jamar, median (IQR) & 11 & $4.0(2.0-8.3)$ & $5.0(2.3-9.7)$ & 0.362 \\
\hline PASS, median (IQR) & 18 & $29.0(23.3-32.8)$ & $\begin{array}{l}33.0(29.5- \\
35.0)\end{array}$ & 0.009 \\
\hline Gait independence score, median (IQR) & 25 & $3.0(1.0-3.0)$ & $3.0(1.5-4.0)$ & 0.024 \\
\hline Pyramidal signs (\%) & 26 & $18(69.2)$ & $16(61.5)$ & 0.560 \\
\hline \multicolumn{5}{|l|}{ Cognitive function } \\
\hline GCS, median (IQR) & 25 & $15.0(14.0-15.0)$ & $\begin{array}{l}15.0(14.0- \\
15.0)\end{array}$ & 1.0 \\
\hline TMT A, mean \pm SD & 9 & $140.9 \pm 72.7$ & $100.1 \pm 47.7$ & 0.015 \\
\hline TMT B, mean \pm SD & 4 & $219.3 \pm 90.9$ & $185.3 \pm 118.5$ & 0.105 \\
\hline Digit span forward, median (IQR) & 22 & $4.0(0.8-6.0)$ & $4.0(0.8-6.3)$ & 0.705 \\
\hline $\begin{array}{l}\text { Regard's 5-point non-verbal fluency test, } \\
\text { mean } \pm \text { SD }\end{array}$ & 20 & $8.8 \pm 7.8$ & $10.8 \pm 8.5$ & 0.037 \\
\hline 12 object naming test, median (IQR) & 25 & $11.5(0-12.0)$ & $11.0(0-12.0)$ & 0.180 \\
\hline Token test errors (language), median (IQR) & 14 & $17.5(0-20.0)$ & $16.0(0-20.0)$ & 0.102 \\
\hline Spatial neglect severity score, median (IQR) & 25 & $1.5(0-3.0)$ & $1.0(0-2.0)$ & 0.031 \\
\hline $\begin{array}{l}\text { a - t-test or Wilcoxon Signed Ranks test, as a } \\
\text { grip strength evaluation with Jamar Hand Dy } \\
\text { Assessment Scale for Stroke; GCS, Glasgow }\end{array}$ & 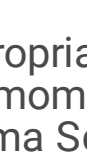 & $\begin{array}{l}\text { Abbreviations: } \\
\text { er; IQR, interqua } \\
\text { le; TMT, Trail M }\end{array}$ & $\begin{array}{l}\text { standard dev } \\
\text { ange; PASS, I } \\
\text { Test. }\end{array}$ & $\begin{array}{l}\text {; Jamar, } \\
\text { ral }\end{array}$ \\
\hline
\end{tabular}

\section{Figures}



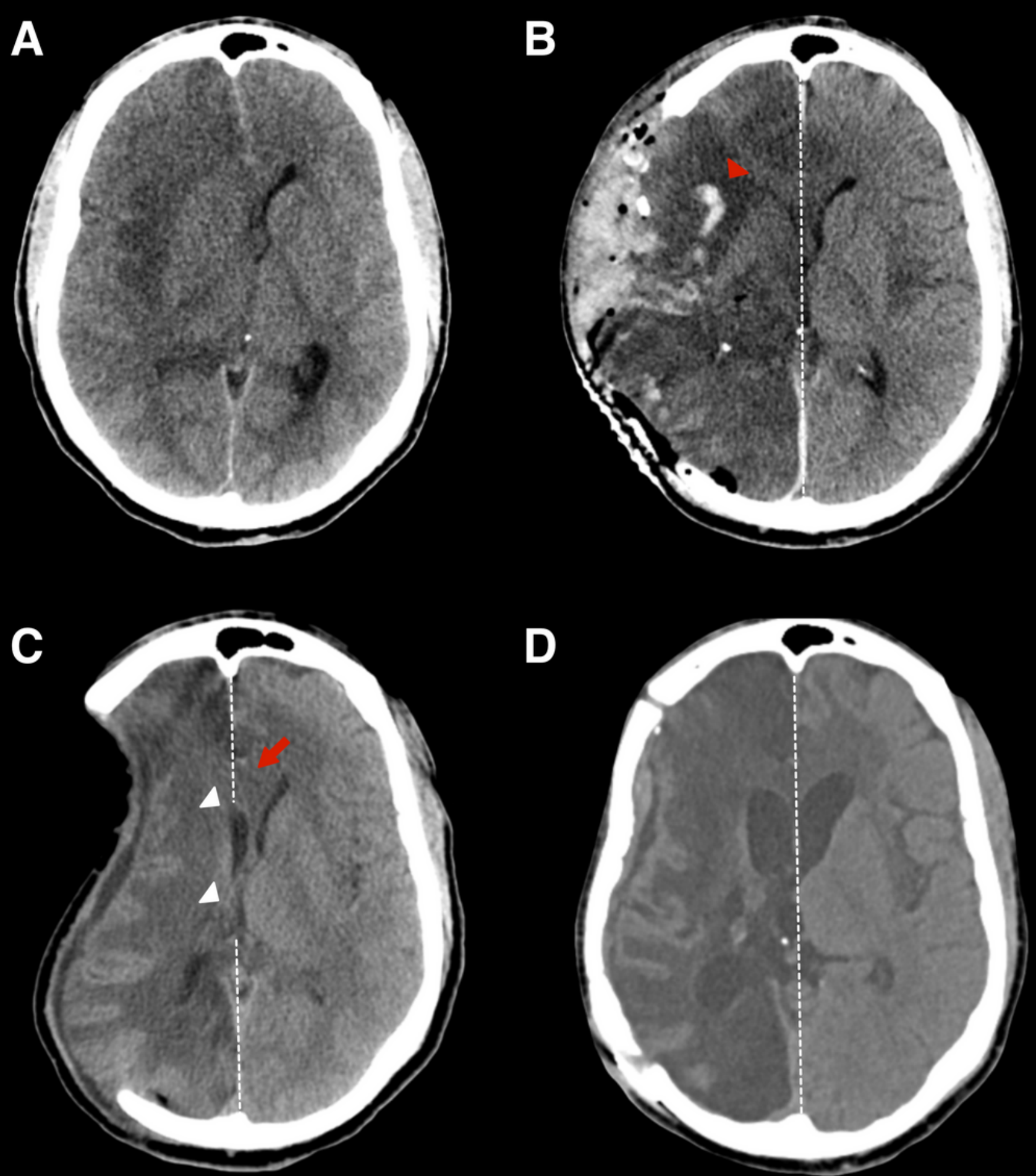

\section{Figure 1}

Serial imaging of SoT. (A) A right-handed individual without previous medical history underwent DC and right temporal lobectomy due to HSV-1 encephalitis induced brain edema. (B) The postoperative CT showed decompression of swollen brain tissue and a new intraparenchymal hemorrhage (red arrowhead). (C) Ten weeks after DC, the patient developed nausea and vomiting, worsening left-sided paresis, aphasia, decreased level of consciousness in a vertical position (GCS decrease from 11 to 8), and 
improvement to baseline in a supine position. Based on these clinical findings, an 'a priori' SoT was diagnosed. CT imaging at ten weeks showed the craniectomy site's sinking appearance, paradoxical deviation of midline structures (red arrow), slit third ventricle, and AHLV (white arrowheads). (D) Cranioplasty was performed at week 11, resolving the radiologic signs of SoT and orthostatism. After cranioplasty, the motor function returned to baseline, consciousness improved (GCS 14), and the patient started to communicate in writing. Abbreviations: DC = decompressive craniectomy; HSV-1 = herpes simplex virus type $1 ; \mathrm{GCS}=$ Glasgow Coma Scale; $\mathrm{AHLV}=$ anterior horn of lateral ventricle; $\mathrm{SoT}=$ syndrome of the trephined.

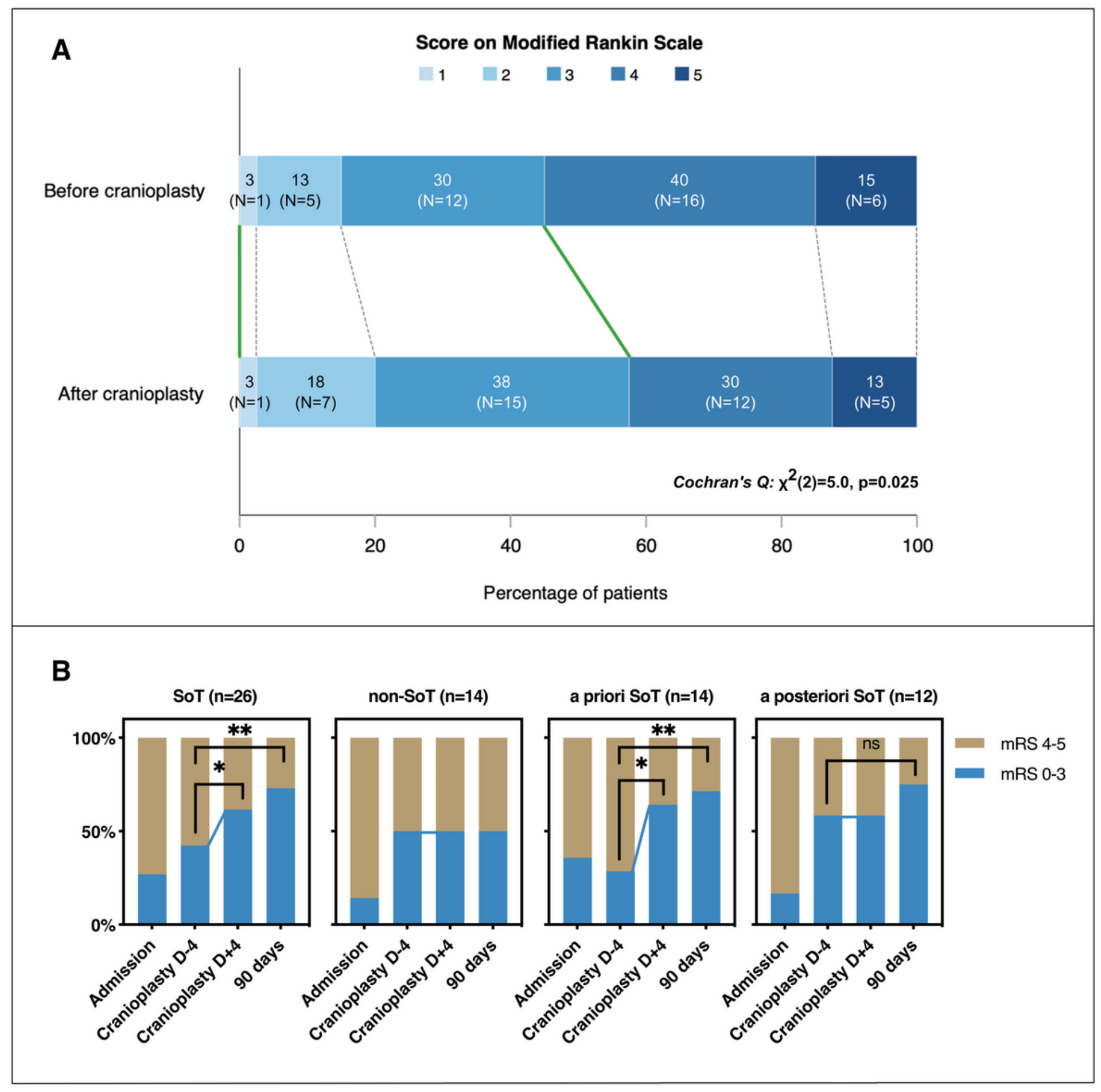




\section{Figure 2}

Impact of cranioplasty on neurological outcome after cranioplasty. (A) Distribution of the score on the modified Rankin scale (mRS) at 1-4 days before and 1-4 days after cranioplasty $(n=40)$. More patients presented a good neurological outcome (mRS 0-3) after the cranioplasty than before $(p=0.025$; green lines). (B) The proportion of good neurological outcome (mRS 0-3) stratified by SoT severity. In the SoT group, there was a significant shift towards a good outcome within four days after the cranioplasty (62\% vs. $42 \%, p=0.025)$ and maintained at 90 days $(73 \%$ vs. $42 \%, p=0.005)$. There was a significant mean mRS improvement in the SoT group within four days after the cranioplasty ( $3.4 \pm 0.9$ vs. $3.7 \pm 0.8, p=$ $0.008)$. In the 'a priori' SoT group, a similar shift towards good outcome ( $64 \%$ vs. $29 \%, p=0.025)$ and improved mRS ( $3.3 \pm 1.0$ vs. $3.9 \pm 0.9, p=0.006)$ was observed. In the 'a posteriori' SoT group, there was no significant change in the mRS within four days after the cranioplasty, but there was a trend towards a good outcome and a significant mean mRS improvement at 90 days (75\% vs. 58\%, $p=0.157$, and $2.9 \pm$ 1.1 vs. $3.4 \pm 0.8, p=0.007$, respectively). Significance level $p<0.05$ (*) and $p<0.01(* *)$. Abbreviations: SoT $=$ syndrome of the trephined; $D-4=$ four days before cranioplasty; $D+4=$ four days after cranioplasty, $n s=$ non-significant. 


\section{Brain swelling resolved SoT Cranioplasty}

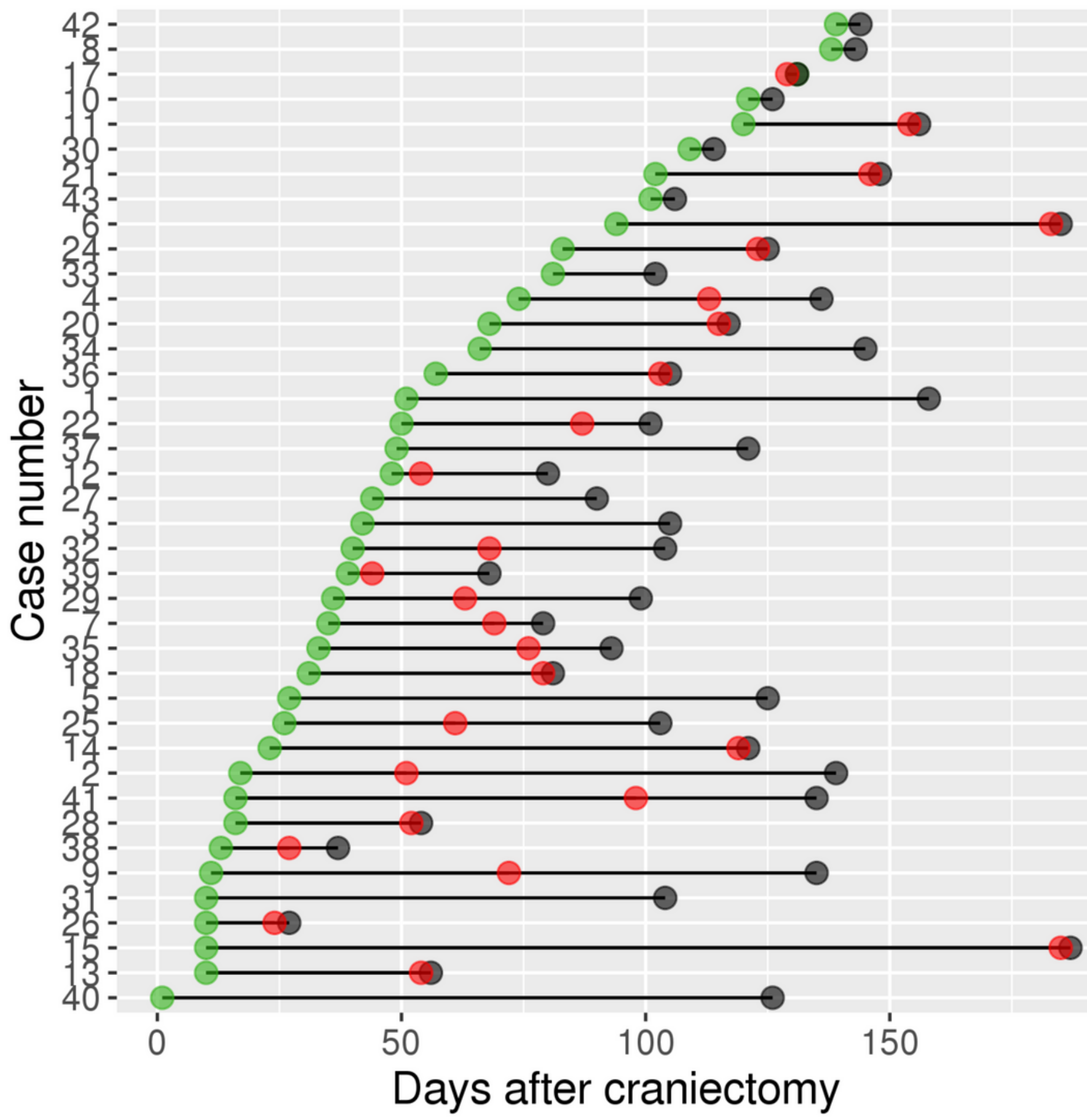

Figure 3

Delay to key events after the craniectomy Days delay to brain swelling disappearance in the craniectomy site (green dot), SoT (red dot), and to cranioplasty (black dot) are indicated for individual patients. The non-overlapping red dots correspond to 'a priori' SoT (clinical deterioration before cranioplasty), and overlapping red and black dots correspond to 'a posteriori' SoT. 'A posteriori' SoT was diagnosed when neurological improvement was observed within four days after the cranioplasty. 


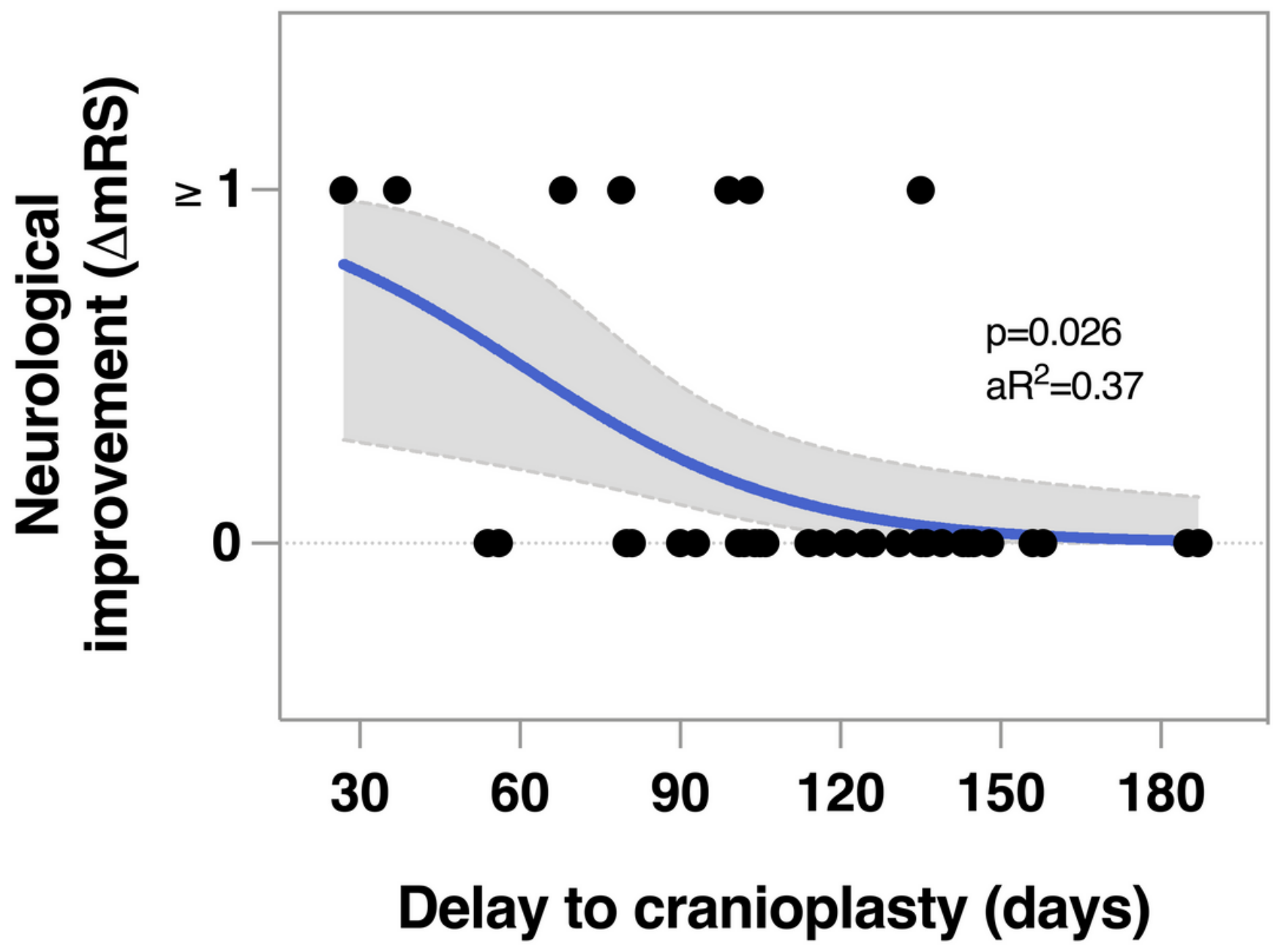

Figure 4

Logistic regression: shorter delay to cranioplasty was associated with better neurological outcomes. Shorter delay to cranioplasty $(\beta=-0.04, p=0.012)$ was associated with improvement in mRS after cranioplasty after adjusting for age and baseline disability. Variance explained by R2 = 0.24 (Cox \& Snell), model $\chi 2(1)=8.62$. Abbreviations: $\mathrm{mRS}=$ modified Rankin Scale, $\mathrm{aR2}=$ adjusted R2.

\section{Supplementary Files}

This is a list of supplementary files associated with this preprint. Click to download.

- STROBEchecklistv4.docx 